\title{
On the Performance of Physically Constrained Multi-Pair Two-Way Massive MIMO Relaying with Zero Forcing
}

\author{
Jun Qian, Christos Masouros, Senior Member, IEEE,
}

\begin{abstract}
In this paper, we consider a physically constrained multi-pair two-way massive multiple-input multipleoutput (MIMO) decode-and-forward (DF) half-duplex relay system, where multiple single-antenna user pairs exchange information through a massive MIMO relay, and we employ zeroforcing reception/zero-forcing transmission (ZFR/ZFT) at the relay. When the number of relay antennas $M$ becomes very large and tends to be infinite, we study the large-scale approximation of the sum spectral efficiency (SE) with the effect of spatial correlation generated by the constrained space. Furthermore, we investigate the energy efficiency (EE) with a practical power consumption model, and demonstrate the impact of the relay antenna number and the size of constrained space on the $\mathbf{E E}$ performance.
\end{abstract}

Index Terms-Decode-and-forward relaying, massive MIMO, spatial correlation, spectral efficiency and energy efficiency, zero forcing processing.

\section{INTRODUCTION}

$\mathbf{M}$ ASSIVE multiple-input multiple-output (MIMO) has become one of the key technologies for the nextgeneration wireless communications towards achieving higher system capacity and data rate demands by serving a great number of users simultaneously [1].

With the application of massive MIMO in the multi-pair relaying system, the proposed system has attracted great attention due to its ability to improve the system capacity, cellular coverage and system throughput, even enhance the service quality for cell edge users [2]-[4]. Originally, oneway relaying system has been studied for multi-pair massive MIMO relaying. Based on the previous studies, one-way relaying might incur spectral efficiency loss [5]. Therefore, two-way relaying is introduced which can improve the spectral efficiency (SE) [6]. To this end, multi-pair two-way relaying is considered where more than one pair of users exchange information, by a shared relay [4], [6]. Generally, the amplify-andforward (AF) protocol is addressed in most previous studies on multi-pair two-way massive MIMO relaying. However, the AF relaying might suffer from noise amplification problem [4]. To this end, decode-and-forward (DF) two-way relaying has been considered as it can achieve better performance than AF scenarios at low signal-to-noise ratios (SNRs) [7]. Moreover,

J. Qian and C. Masouros are with the Department of Electronic and Electrical Engineering, University College London, London WC1E 7JE, U.K. (email: jun.qian.15@ucl.ac.uk; c.masouros@ucl.ac.uk).

This work was supported by the Engineering and Physical Sciences Research Council (EPSRC) project EP/M014150/1.
DF two-way relaying has the potential to perform separate linear precoding on each relaying communication direction [4].

In the light of the above, we investigate a multi-pair twoway half-duplex DF massive MIMO relaying system with imperfect channel state information (CSI) and zero-forcing (ZF) processing. Moreover, when employing large antenna arrays in constrained physical space, the spatial correlation becomes determinant for the resulting performance [8]-[10], the consideration of spatial correlation is crucial in the multipair relaying system. In this case, based on the studies in [4], a detailed analysis of the achievable sum SE of a physically constrained system is presented and we study a practical power consumption model to analyze the energy efficiency (EE) of the proposed relaying system with respect to spatial correlation.

Notation: We use $\mathbf{H}^{T}, \mathbf{H}^{H}, \mathbf{H}^{*}$ and $\mathbf{H}^{-1}$ to represent the transpose, conjugate-transpose, conjugate and the inverse of matrix $\mathbf{H}$, respectively. Moreover, $\mathbf{I}_{M}$ is an $M \times M$ identity matrix. In addition, $\|\cdot\|$ and $\|\cdot\|_{F}$ denotes the Euclidian from and Frobenius form, respectively. Finally, $\mathbb{E}\{\cdot\}$ is the expectation operator and $\operatorname{var}\{\cdot\}$ is the variance operator.

\section{System Model}

\section{A. Channel Model}

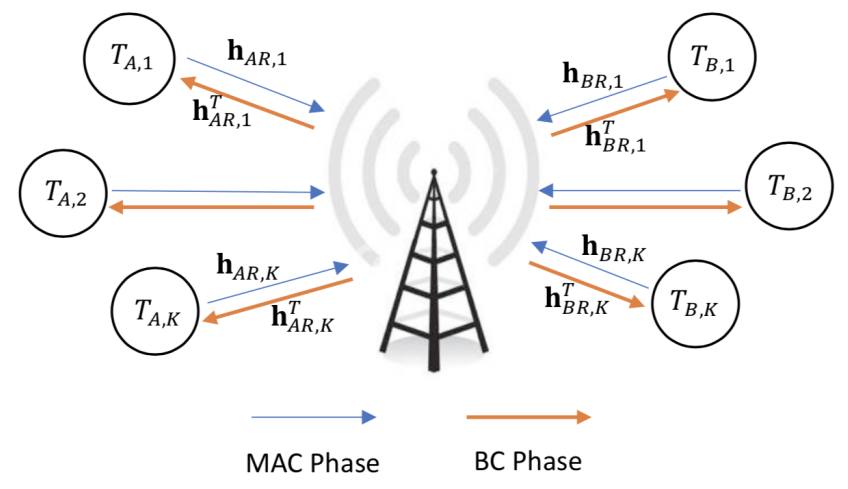

Fig. 1. Multi-pair two-way DF relaying system.

The proposed physically-constrained multi-pair two-way half-duplex DF relaying system is shown in Fig. 1, where $K$ pairs of single-antenna users, defined as $\mathrm{T}_{A, i}$ and $\mathrm{T}_{B, i}, i=$ $1, \ldots, K$, can exchange information with each other through the relay $\mathrm{T}_{R}$ with $M$ closely-spaced antennas, while $M \gg K$. 
Moreover, there is no direct link between user pairs as assumption. Generally, massive MIMO system is assumed to be performed in a time-division duplexing (TDD) mode [11], [12] while in the same frequency band [13]. Therefore, we assume that the proposed system is modeled as spatially-correlated Rayleigh fading under TDD protocol and channel reciprocity works. To this end, the uplink and downlink channels between $\mathrm{T}_{X, i}, X=A, B$ and $\mathrm{T}_{R}$ are defined as $\mathbf{h}_{X R, i}$ and $\mathbf{h}_{X R, i}^{T}, i=1, \ldots, K$, respectively. The relevant uplink propagation channel can be expressed as [9], [14]

$$
\mathbf{h}_{X R, i}=\mathbf{A}_{i}^{H} \mathbf{g}_{X R, i},
$$

where $\mathbf{g}_{X R, i} \sim \operatorname{CN}\left(0, \beta_{X R, i} \mathbf{I}_{D_{i}}\right), D_{i}$ represents the number of signal propagation paths with different angles of departure, $\beta_{A R, i}$ and $\beta_{B R, i}$ represent the large-scale effect which are assumed as constants in this paper for simplicity, and $\mathbf{A}_{i}$ $\in \mathbb{C}^{D_{i} \times M}$ is the transmit steering matrix at $T_{X, i}$ given by [15], [16]

$$
\mathbf{A}_{i}=\frac{1}{\sqrt{D_{i}}}\left[\mathbf{a}^{\mathrm{T}}\left(\theta_{i, 1}\right), \ldots, \mathbf{a}^{\mathrm{T}}\left(\theta_{i, D_{i}}\right)\right]^{\mathrm{T}} .
$$

here, $\theta_{i, k}, k=1, \ldots, D_{i}$ represents the spreading angle describing the directions of departure. The corresponding steering vectors a for the uniform linear array (ULA) topology are in terms of [9]

$$
\mathbf{a}\left(\theta_{i, k}\right)=\left[1, e^{j 2 \pi d \sin \left(\theta_{i, k}\right)}, \ldots, e^{j 2 \pi(M-1) d \sin \left(\theta_{i, k}\right)}\right],
$$

where $d=\frac{D_{\text {total }}}{M-1}$ is the inter-antenna distance with $D_{\text {total }}$, the total spacing length normalized by the carrier wavelength $\lambda$. We assume that there is no receive correlation since the interantenna distance is greater than $\lambda$ [9]. Additionally, the channel matrix can be formed as $\mathbf{H}_{X R}=\left[\mathbf{h}_{X R, 1}, \ldots, \mathbf{h}_{X R, K}\right] \in \mathbb{C}^{M \times K}$, $X=A, B$.

For the proposed relaying system, the data transmission process are divided into two phases with equal time slots. Generally, this two-phase protocol is called the Multiple Access Broadcast (MABC) protocol [7]. In the first MAC phase, all users transmit their respective signals to the relay simultaneously. Therefore, the received signal at the relay can be expressed as [4], [17]

$$
\mathbf{y}_{r}=\sum_{i=1}^{K}\left(\sqrt{p_{A, i}} \mathbf{h}_{A R, i} s_{A R, i}+\sqrt{p_{B, i}} \mathbf{h}_{B R, i} s_{B R, i}\right)+\mathbf{n}_{R},
$$

where $s_{X R, i}$ is the Gaussian signal with zero mean and unit power transmitted by the $i$-th user $T_{X, i}, p_{X, i}$ is the average transmit power of $\mathrm{T}_{X, i}, X=A, B . \mathbf{n}_{R}$ is a vector of additive white Gaussian noise (AWGN) at the relay whose elements satisfying (i.i.d) $\mathrm{CN}(0,1)$. For low-complexity transmission, linear processing is applied at the relay and the transformed signal can be obtained as

$$
\mathbf{z}_{r}=\mathbf{F}_{M A C} \mathbf{y}_{r}
$$

here, $\mathbf{F}_{M A C} \in \mathbb{C}^{2 K \times M}$ is the linear receiver matrix in the MAC phase.

In the second $\mathrm{BC}$ phase, the relay decodes the received information and then re-encodes and broadcasts it to users [4].
The linear precoding matrix $\mathbf{F}_{B C} \in \mathbb{C}^{M \times 2 K}$ in the BC phase is applied to obtain the transmit signal of the relay as

$$
\mathbf{y}_{t}=\rho_{D F} \mathbf{F}_{B C} \mathbf{s}
$$

where $\mathbf{s}=\left[\mathbf{s}_{A}^{T}, \mathbf{s}_{B}^{T}\right]^{T}$ denotes the decoded signal and $\rho_{D F}$ specified in the following, is the normalization coefficient determined by the average relay power constraint $\mathbb{E}\left\{\left\|\mathbf{y}_{t}\right\|^{2}\right\}=p_{r}$. In this case, the received signals at $\mathrm{T}_{X, i}, X=A, B$ can be given by

$$
z_{X, i}=\mathbf{h}_{X R, i}^{T} \mathbf{y}_{t}+n_{X, i},
$$

with the standard AWGN at $T_{X, i}, n_{X, i} \sim \mathcal{C N}(0,1), X=A, B$.

\section{B. Linear Processing and Channel Estimation}

Generally, both inter-pair interference and inter-user interference can be eliminated by linear processing in massive MIMO system [18]. In order to achieve low-complexity transmission, a basic linear processing scheme, $\mathrm{ZF}$ processing is applied at the relay. Therefore, the linear processing matrix $\mathbf{F}_{M A C} \in \mathbb{C}^{2 K \times M}$ and $\mathbf{F}_{B C} \in \mathbb{C}^{M \times 2 K}$ given above can be defined as

$$
\begin{gathered}
\mathbf{F}_{M A C}=\left(\left[\hat{\mathbf{H}}_{A R}, \hat{\mathbf{H}}_{B R}\right]^{H}\left[\hat{\mathbf{H}}_{A R}, \hat{\mathbf{H}}_{B R}\right]\right)^{-1}\left[\hat{\mathbf{H}}_{A R}, \hat{\mathbf{H}}_{B R}\right]^{H}, \\
\mathbf{F}_{B C}=\left[\hat{\mathbf{H}}_{B R}, \hat{\mathbf{H}}_{A R}\right]^{*}\left(\left[\hat{\mathbf{H}}_{B R}, \hat{\mathbf{H}}_{A R}\right]^{T}\left[\hat{\mathbf{H}}_{B R}, \hat{\mathbf{H}}_{A R}\right]^{*}\right)^{-1},
\end{gathered}
$$

respectively. In (8)-(9) above, $\hat{\mathbf{H}}_{X R}$ are the CSI estimates of the respective channels, $X=A, B$. To simplify the expressions in the following sections, we define that $\mathbf{F}_{M A C}^{A R} \in \mathbb{C}^{K \times M}, \mathbf{F}_{M A C}^{B R} \in$ $\mathbb{C}^{K \times M}$ representing the first $K$ rows and the rest $K$ rows of $\mathbf{F}_{M A C}$, respectively. Meanwhile, $\mathbf{F}_{B C}^{R B} \in \mathbb{C}^{M \times K}, \mathbf{F}_{B C}^{R A} \in \mathbb{C}^{M \times K}$ are the first $K$ columns of and the rest $K$ columns of $\mathbf{F}_{B C}$, respectively.

Moreover, imperfect CSI is employed in this paper. In TDD systems, transmitting pilots is the basic way to estimate channels at the relay [4], [15]. We assume that the channels are estimated by using the minimum mean square error (MMSE) estimator [3], [15], [19]. Thus, we can have the channel estimates as

$$
\mathbf{h}_{X R, i}=\hat{\mathbf{h}}_{X R, i}+\mathbf{e}_{X R, i}=\mathbf{A}_{i}^{H} \hat{\mathbf{g}}_{A R, i}+\mathbf{A}_{i}^{H} \mathbf{q}_{A R, i},
$$

where $\hat{\mathbf{h}}_{X R, i}$ and $\mathbf{e}_{X R, i}$ are the $i$-th columns of the estimated matrices $\hat{\mathbf{H}}_{X R}$ and estimation error matrices $\mathbf{E}_{X R}, X=A, B$, respectively. The elements in $\hat{\mathbf{g}}_{X R, i}$ and $\mathbf{q}_{X R, i}$ are Gaussian random variables with zero mean, variance $\sigma_{X R, i}^{2}=\frac{\tau_{p} p_{p} \beta_{X R, i}^{2}}{1+\tau_{p} p_{p} \beta_{X R, i}}$ and $\tilde{\sigma}_{X R, i}^{2}=\frac{\beta_{X R, i}}{1+\tau_{p} p_{p} \beta_{X R, i}}, X=A, B$, respectively [4].

\section{Performance Analysis}

\section{A. Exact results of Spectral Efficiency}

In this section, we focus on the spectral efficiency (SE) of the proposed system. To avoid repetition, we refer the reader to [4] for a more systematic study of the process of calculating the SE.

In the MAC phase, via applying the linear processing matrix $\mathbf{F}_{M A C}$ to the received signals, the transformed signals can be obtained by (5). Therefore, the interference power due to 
estimation error, inter-user interference power and compound noise power of the post-processing signals $\mathbf{z}_{r}$ for the $i$-th pair of users can be obtained as

$$
\begin{aligned}
A_{i}= & p_{A, i}\left(\left|\mathbf{F}_{M A C, i}^{A R} \mathbf{e}_{A R, i}\right|^{2}+\left|\mathbf{F}_{M A C, i}^{B R} \mathbf{e}_{A R, i}\right|^{2}\right) \\
& +p_{B, i}\left(\left|\mathbf{F}_{M A C, i}^{A R} \mathbf{e}_{B R, i}\right|^{2}+\left|\mathbf{F}_{M A C, i}^{B R} \mathbf{e}_{B R, i}\right|^{2}\right), \\
B_{i}= & \sum_{j \neq i} p_{A, j}\left(\left|\mathbf{F}_{M A C, i}^{A R} \mathbf{h}_{A R, j}\right|^{2}+\left|\mathbf{F}_{M A C, i}^{B R} \mathbf{h}_{A R, j}\right|^{2}\right) \\
& +\sum_{j \neq i} p_{B, j}\left(\left|\mathbf{F}_{M A C, i}^{A R} \mathbf{h}_{B R, j}\right|^{2}+\left|\mathbf{F}_{M A C, i}^{B R} \mathbf{h}_{B R, j}\right|^{2}\right), \\
C_{i}= & \left\|\mathbf{F}_{M A C, i}^{A R}\right\|^{2}+\left\|\mathbf{F}_{M A C, i}^{B R}\right\|^{2},
\end{aligned}
$$

respectively. With the expressions of desired signals, the $\mathrm{SINR}_{X R, i}, X=A, B$, can be given by

$$
\operatorname{SINR}_{X R, i}=\frac{p_{X, i}\left(\left|\mathbf{F}_{M A C, i}^{A R} \hat{\mathbf{h}}_{X R, i}\right|^{2}+\left|\mathbf{F}_{M A C, i}^{B R} \hat{\mathbf{h}}_{X R, i}\right|^{2}\right)}{A_{i}+B_{i}+C_{i}} .
$$

Moreover, we consider the standard lower capacity bound associated with the worst-case uncorrelated additive noise [4]; therefore, the sum achievable SE of the $i$-th user pair in the MAC phase is given by (15) on the bottom of this page and the SE of the user $T_{X, i}$ to the relay, $X=A, B$, can be denoted as

$$
R_{X R, i}=\frac{\tau_{c}-\tau_{p}}{2 \tau_{c}} \times E\left\{\log _{2}\left(1+\operatorname{SINR}_{X R, i}\right)\right\}
$$

In the BC phase, with $\mathbf{F}_{B C}$ applied to generate the relay's transmit signal, the received signals at $T_{X, i}$ can be calculated by (6), and we can obtain the SE of the relay to the $i$-th user $T_{X, i}, X=A, B$,

$$
R_{R X, i}=\frac{\tau_{c}-\tau_{p}}{2 \tau_{c}} \times E\left\{\log _{2}\left(1+\operatorname{SINR}_{R X, i}\right)\right\},
$$

where $\operatorname{SINR}_{R X, i}$ is given by (18), shown on the bottom of this page. Furthermore, the sum SE of the $i$-th user pair in the BC phase is defined as the sum of the end-to-end SE from $T_{A, i}$ to $T_{B, i}$ and the end-to-end SE from $T_{B, i}$ to $T_{A, i}$,

$$
R_{2, i}=\min \left(R_{A R, i}, R_{R B, i}\right)+\min \left(R_{B R, i}, R_{R A, i}\right) .
$$

According to [4], [20], the sum SE of the multi-pair two-way DF relaying system can be given by

$$
R=\sum_{i=1}^{K} R_{i}=\sum_{i=1}^{K} \min \left(R_{1, i}, R_{2, i}\right)
$$

\section{B. Approximations of Spectral Efficiency}

In practical, the large-scale approximation of the SE for the $i$-th user pair can be derived when the relay employs a great number of antennas, i.e., $M \rightarrow \infty$.

Lemma 1: When $M \rightarrow \infty, \hat{\mathbf{G}}_{X R}=\left[\hat{\mathbf{g}}_{X R, 1}, \ldots, \hat{\mathbf{g}}_{X R, K}\right] \in \mathbb{C}^{M \times D}$, $X=A, B$ and to simplify the model, we consider $D_{i}=D$ with $i=1, \ldots, K$. In this case, due to the uncorrelation of any two columns in the estimated matrix $\hat{\mathbf{G}}_{X R}, X=A, B$, the inner product of any two columns can be defined as [2], [21]

$$
\frac{1}{M} \cdot \hat{\mathbf{g}}_{X R, i}^{H} \hat{\mathbf{g}}_{X R, j} \rightarrow\left\{\begin{array}{ll}
\sigma_{X R, i}^{2}, & i=j \\
0, & i \neq j
\end{array} .\right.
$$

With increasing relay antenna number, the vectors in $\hat{\mathbf{G}}_{X R}$ become asymptotically mutually orthogonal. Therefore, $\hat{\mathbf{G}}_{X R}^{H} \hat{\mathbf{G}}_{X R}$ can be assumed to approach a diagonal matrix [8]. With the assistance of Lemma 1, the linear processing matrices $\mathbf{F}_{M A C}$ and $\mathbf{F}_{B C}$ can be simplified when $M \rightarrow \infty$ as follows

$$
\begin{gathered}
\mathbf{F}_{M A C} \rightarrow\left[\begin{array}{c}
\left(\hat{\mathbf{H}}_{A R}^{H} \hat{\mathbf{H}}_{A R}\right)^{-1} \hat{\mathbf{H}}_{A R}^{H} \\
\left(\hat{\mathbf{H}}_{B R}^{H} \hat{\mathbf{H}}_{B R}\right)^{-1} \hat{\mathbf{H}}_{B R}^{H}
\end{array}\right], \\
\mathbf{F}_{B C} \rightarrow\left[\hat{\mathbf{H}}_{B R}^{*}\left(\hat{\mathbf{H}}_{B R}^{T} \hat{\mathbf{H}}_{B R}^{*}\right)^{-1}, \hat{\mathbf{H}}_{A R}^{*}\left(\hat{\mathbf{H}}_{A R}^{T} \hat{\mathbf{H}}_{A R}^{*}\right)^{-1}\right],
\end{gathered}
$$

respectively. The normalization coefficient $\rho_{D F}$ can be expressed as

$$
\rho_{D F}^{Z F}=\sqrt{\frac{p_{r}}{E\left\{\left\|\mathbf{F}_{B C}\right\|_{\mathrm{F}}^{2}\right\}}}=\sqrt{\frac{M^{2} \cdot p_{r}}{\sum_{i=1}^{K}\left(\frac{1}{\sigma_{A R, i}^{2}}+\frac{1}{\sigma_{B R, i}^{2}}\right) \sum_{m=1}^{D} \sum_{n=1}^{M}\left|\mathbf{A}_{m, n}\right|^{2}}} .
$$

In the line with above, considering the DF protocol and the properties of $\mathrm{ZF}$ processing [22], when $M \rightarrow \infty$, the approximations associated with $\hat{R}_{i}\left(R_{i}-\hat{R}_{i} \rightarrow 0\right)$ can be given by

$$
\hat{R}=\sum_{i=1}^{K} \hat{R}_{i}=\sum_{i=1}^{K} \min \left(\hat{R}_{1, i}, \hat{R}_{2, i}\right)
$$

The approximations of the achievable SE in the MAC and BC phases, and the SE of the user pair/relay to the relay/user pair can be given by (27)-(29) on the top of next page while

$$
\hat{R}_{2, i}=\min \left(\hat{R}_{A R, i}, \hat{R}_{R B, i}\right)+\min \left(\hat{R}_{B R, i}, \hat{R}_{R A, i}\right),
$$

Here, $\Theta_{i}=\mathbf{A}_{i} \mathbf{A}_{i}^{\mathrm{H}}$ with the assumption that $\mathbf{A}_{i}=\mathbf{A}_{j}, i, j=$ $1, \ldots, K$. Based on (28)-(29), $\hat{R}_{B R, i}$ and $\hat{R}_{R B, i}$ can be obtained by using the transmit powers $p_{B, i}, p_{A, i}$, and the subscripts "BR", "AR" to replace the transmit powers $p_{A, i}, p_{B, i}$, and the subscripts "AR", "BR" in $\hat{R}_{A R, i}$ and $\hat{R}_{R A, i}$, respectively.

$$
\begin{gathered}
R_{1, i}=\frac{\tau_{c}-\tau_{p}}{2 \tau_{c}} \times E\left\{\log _{2}\left(1+\frac{p_{A, i}\left(\left|\mathbf{F}_{M A C, i}^{A R} \hat{\mathbf{h}}_{A R, i}\right|^{2}+\left|\mathbf{F}_{M A C, i}^{B R} \hat{\mathbf{h}}_{A R, i}\right|^{2}\right)+p_{B, i}\left(\left|\mathbf{F}_{M A C, i}^{A R} \hat{\mathbf{h}}_{B R, i}\right|^{2}+\left|\mathbf{F}_{M A C, i}^{B R} \hat{\mathbf{h}}_{B R, i}\right|^{2}\right)}{A_{i}+B_{i}+C_{i}}\right)\right\} . \\
\operatorname{SINR}_{R X, i}=\frac{\left|\hat{\mathbf{h}}_{X R, i}^{T} \mathbf{F}_{B C, i}^{R X}\right|^{2}}{\left|\mathbf{e}_{X R, i}^{T} \mathbf{F}_{B C, i}^{R X}\right|^{2}+\sum_{j=1}^{K}\left(\left|\mathbf{h}_{X R, i}^{T} \mathbf{F}_{B C, j}^{R A}\right|^{2}+\left|\mathbf{h}_{X R, i}^{T} \mathbf{F}_{B C, j}^{R B}\right|^{2}\right)-\left|\mathbf{h}_{X R, i}^{T} \mathbf{F}_{B C, i}^{R X}\right|^{2}+\frac{1}{\rho_{D F}^{2}}} .
\end{gathered}
$$


$\hat{R}_{1, i}^{Z F}=\frac{\tau_{c}-\tau_{p}}{2 \tau_{c}} \log _{2}\left(1+\frac{p_{A, i}+p_{B, i}}{\sum_{j=1}^{K} p_{A, j}\left(\frac{\tilde{\sigma}_{A R, j}^{2}}{M^{2} \sigma_{A R, i}^{2}}+\frac{\tilde{\sigma}_{A R, j}^{2}}{M^{2} \sigma_{B R, i}^{2}}\right) \sum_{m=1}^{D} \sum_{n=1}^{D}\left|\Theta_{m, n}\right|^{2}+\sum_{j=1}^{K} p_{B, j}\left(\frac{\tilde{\sigma}_{B R, j}^{2}}{M^{2} \sigma_{A R, i}^{2}}+\frac{\tilde{\sigma}_{B R, j}^{2}}{M^{2} \sigma_{B R, i}^{2}}\right) \sum_{m=1}^{D} \sum_{n=1}^{D}\left|\Theta_{m, n}\right|^{2}+\left(\frac{1}{M \sigma_{A R, i}^{2}}+\frac{1}{M \sigma_{B R, i}^{2}}\right)}\right)$,

$$
\begin{gathered}
\hat{R}_{A R, i}^{Z F}=\frac{\tau_{c}-\tau_{p}}{2 \tau_{c}} \log _{2}\left(1+\frac{p_{A, i}}{\sum_{j=1}^{K} p_{A, j}\left(\frac{\tilde{\sigma}_{A R, j}^{2}}{M^{2} \sigma_{A R, i}^{2}}+\frac{\tilde{\sigma}_{A R, j}^{2}}{M^{2} \sigma_{B R, i}^{2}}\right) \sum_{m=1}^{D} \sum_{n=1}^{D}\left|\Theta_{m, n}\right|^{2}+\sum_{j=1}^{K} p_{B, j}\left(\frac{\tilde{\sigma}_{B R, j}^{2}}{M^{2} \sigma_{A R, i}^{2}}+\frac{\tilde{\sigma}_{B R, j}^{2}}{M^{2} \sigma_{B R, i}^{2}}\right) \sum_{m=1}^{D} \sum_{n=1}^{D}\left|\Theta_{m, n}\right|^{2}+\left(\frac{1}{M \sigma_{A R, i}^{2}}+\frac{1}{M \sigma_{B R, i}^{2}}\right)}\right), \\
\hat{R}_{R A, i}^{Z F}=\frac{\tau_{c}-\tau_{p}}{2 \tau_{c}} \log _{2}\left(1+\frac{M^{2} p_{r}}{p_{r} \sum_{j=1}^{K}\left(\frac{\tilde{\sigma}_{A R, i}^{2}}{\sigma_{A R, j}^{2}}+\frac{\beta_{A R, i}}{\sigma_{B R, j}^{2}}\right) \sum_{m=1}^{D} \sum_{n=1}^{D}\left|\Theta_{m, n}\right|^{2}+\sum_{j=1}^{K}\left(\frac{1}{\sigma_{A R, j}^{2}}+\frac{1}{\sigma_{B R, j}^{2}}\right) \sum_{m=1}^{M} \sum_{n=1}^{D}\left|A_{m, n}\right|^{2}}\right),
\end{gathered}
$$

\section{Energy Efficiency}

Generally, the energy efficiency is the ratio of the achievable sum SE to the total system power consumption [18]. Then, the system energy efficiency is defined as [2], [15], [18]

$$
\epsilon=\frac{R}{P_{\text {total }}}
$$

where $R$ denotes the sum SE defined in (20), $P_{\text {total }}$ represents the total power consumption. In practical terms, the total power consumption consists of the transmit signal power, the powers of the operating static circuits and the RF components in each $\mathrm{RF}$ chain, while each antenna is connected to one RF chain [18]. Thus, the power consumption model of the users and the relay in this proposed system can be given by [15], [17],

$$
\begin{gathered}
P_{t o t, i}=\frac{1}{2 \tau_{c}}\left[\frac{\left(\tau_{c}-\tau_{p}\right) p_{u}+\tau_{p} p_{p}}{\zeta_{i}}+\tau_{c} \cdot P_{R F, i}\right] \\
=\frac{1}{2}\left[\frac{\left(\tau_{c}-\tau_{p}\right) p_{u}+\tau_{p} p_{p}}{\tau_{c} \zeta_{i}}+\left(P_{D A C, i}+P_{m i x, i}+P_{f i l t, i}+P_{s y n, i}\right)\right] \\
P_{t o t, r}=\frac{1}{2 \tau_{c}}\left[\frac{\tau_{c} p_{r}}{\zeta_{r}}+\tau_{c} \cdot P_{R F, r}\right] \\
=\frac{1}{2}\left[\frac{p_{r}}{\zeta_{r}}+M\left(P_{D A C, r}+P_{\text {mix }, r}+P_{f i l t, r}\right)+P_{s y n, r}\right]
\end{gathered}
$$

respectively. Here, $P_{t o t, i}$ is the total power of $i$-th user and $P_{t o t, r}$ is the total power of the relay. $\zeta_{i}$ and $\zeta_{r}$ represents the power amplifier efficiency of the $i$-th user and the relay, respectively. $P_{s y n}$ is the power consumption of the frequency synthesizer, $P_{D A C}, P_{m i x}$ and $P_{\text {filt }}$ represent the power of the digital-to-analog converters (DACs), signal mixers and filters respectively [15]. In this case, the total power consumption $P_{\text {total }}$ for the system is

$$
P_{\text {total }}=2 K \cdot P_{t o t, i}+P_{t o t, r}+P_{\text {static }}
$$

while $P_{\text {static }}$ is the power of all the static circuits [18]. In order to simplify the power consumption model in the simulation, we assume that $\zeta_{i}=\zeta_{r}=\zeta, P_{D A C, i}=P_{D A C, r}=P_{D A C}, P_{m i x, i}=$ $P_{\text {mix }, r}=P_{\text {mix }}, P_{\text {filt }, i}=P_{\text {filt }, r}=P_{\text {filt }}$ and $P_{\text {syn }, i}=P_{\text {syn }, r}=P_{\text {syn }}$ for $i=1,2, \ldots, K$.

\section{Numerical Results}

In this section, simulation results are presented to validate the analysis for the performance of SE and EE of the physically constrained multi-pair two-way half-duplex DF relaying system. Generally, the following parameters are employed in the simulation. According to the LTE standard, the coherence interval length is $\tau_{c}=196$ (symbols) and the pilot sequence length is $\tau_{p}=2 \mathrm{~K}$ which is the minimum requirement. For simplicity, we assume that the large-scale fading parameters $\beta_{A R, i}=\beta_{B R, i}=1$. For the power consumption model, we consider that user transmit power $p_{A, i}=p_{B, i}=p_{u}, i=1, \ldots, K$, $P_{D A C}=7.8 \mathrm{~mW}, P_{\text {mix }}=15.2 \mathrm{~mW}, P_{\text {filt }}=10 \mathrm{~mW}, P_{\text {syn }}=25 \mathrm{~mW}$ and $P_{\text {static }}=2 \mathrm{~W}[15]$.

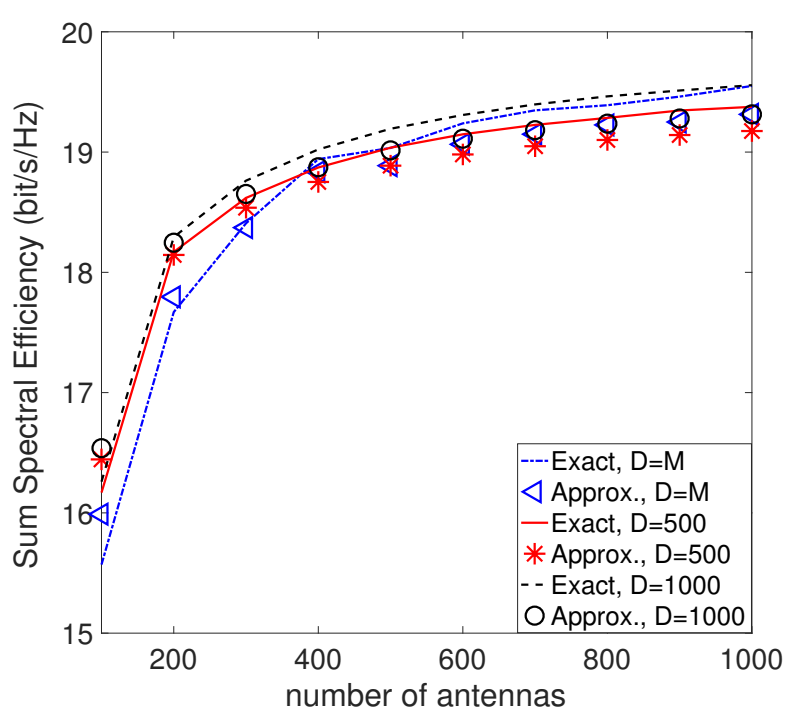

Fig. 2. Sum SE v.s. number of relay antennas $M$ for $K=5, p_{p}=p_{u}=5 \mathrm{~dB}$ and $p_{r}=10 \mathrm{~dB}$.

Fig. 2 shows the sum SE vs the number of relay antennas $M$ with different settings of the signal propagation path number $D$. Note that the "Approx." (Approximations) are obtained by 
applying (25)-(29), and the "Exact" (Numerical results) are generated according to (11)-(20). We can observe that the sum SE grows unbounded with respect to $M$, Moreover, larger number of $D$ can help to achieve better sum SE, especially when $M$ is small.

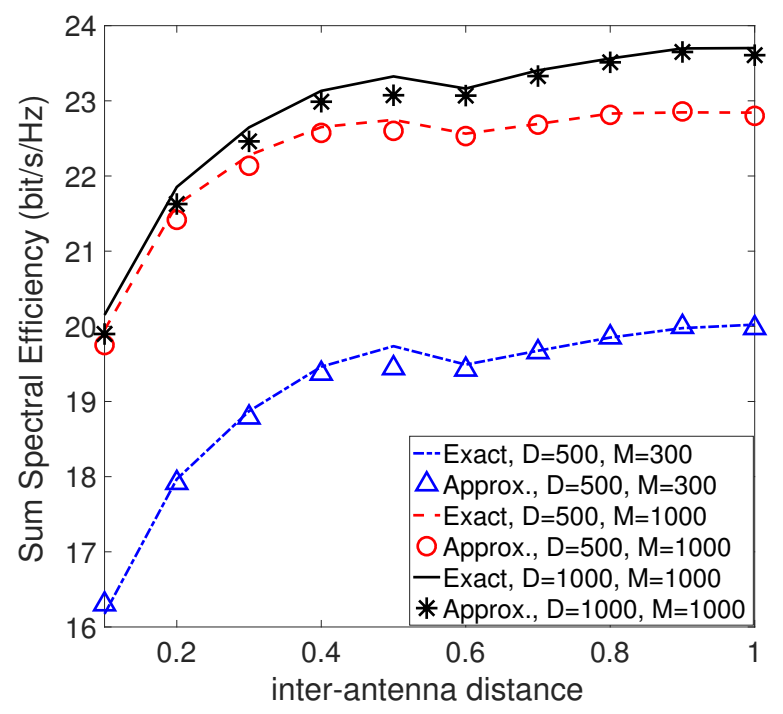

Fig. 3. Sum SE v.s. inter-antenna distance for $K=5, p_{p}=p_{u}=5 \mathrm{~dB}$ and $p_{r}=10 \mathrm{~dB}$.

As shown in Fig.3, simulation results are presented to display the relationship between the sum SE and the interantenna distance of the proposed physically constrained system. Similar with Fig.2, larger $D$ can help to achieve a better SE performance; moreover, $d=0.5$ normalized by the carrier wavelength $\lambda$ can be selected to make better use of spatial correlation to achieve greater sum SE while antennas are closely spaced.

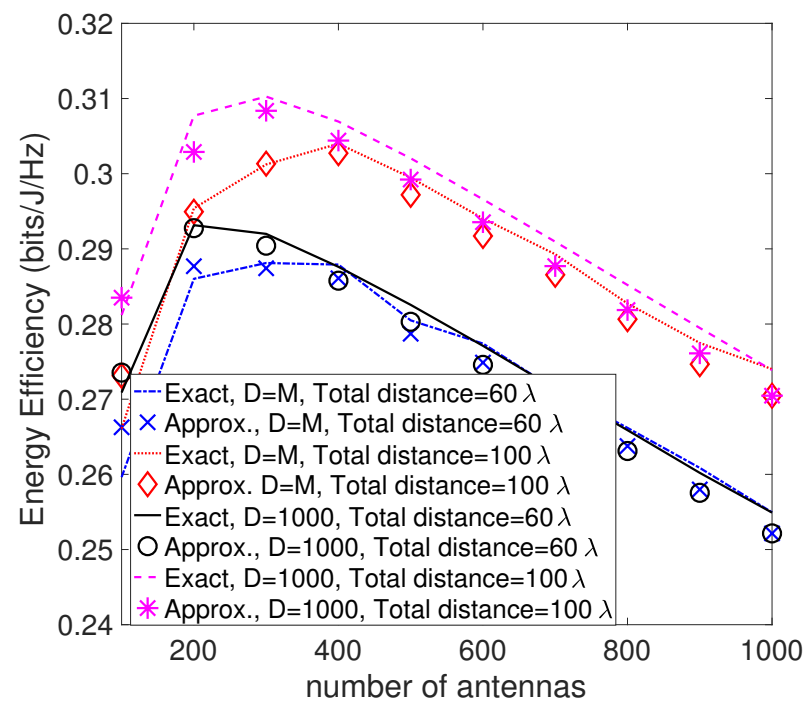

Fig. 4. EE v.s. number of relay antennas $M$ for $K=5, p_{p}=p_{u}=5 \mathrm{~dB}$ and $p_{r}=10 \mathrm{~dB}$.
Fig. 4 represents the relationship between $\mathrm{EE}$ and the number of antennas $M$. It is clearly shown that after achieving an optimal EE performance, EE would decrease with respect to $M$, and larger $D$ has a positive effect on EE when $M$ is small. Moreover, we can observe that when $D_{\text {total }}=100$ normalized by the carrier wavelength $\lambda$, the optimal EE can be achieved when $M=300$ while $d=D_{\text {total }} / M=\frac{1}{3}$, similarly, when $D_{\text {total }}=60$, the optimal EE is achieved when $M=200$ with $d=D_{\text {total }} / M=0.3$. In this specific case we considered here, $d \approx 0.3$ can be determined to achieve an optimal EE in the proposed physically spaced system.

\section{Conclusion}

In this paper, we have studied the sum SE and EE performance of a physically constrained multi-pair two-way halfduplex DF relaying system with ZF processing and imperfect CSI. Particularly, a closed-form large-scale approximation of the SE is investigated with the effect of spatial correlation between closely spaced relay antennas. With the assistance of the approximations, the relationships between the Sum SE with number of antennas and the inter-antenna distance are studied. Moreover, a practical power consumption model is characterized to study the system EE which can benefit from the spatial correlation to achieve an optimal EE performance with an optimal inter-antenna distance in the physically constrained system.

\section{REFERENCES}

[1] F. Rusek, D. Persson, B. K. Lau, E. G. Larsson, T. L. Marzetta, O. Edfors, and F. Tufvesson, "Scaling up MIMO: Opportunities and challenges with very large arrays," IEEE Signal Process. Mag., vol. 30, no. 1, pp. 40-60, Jan 2013.

[2] X. Sun, K. Xu, W. Ma, and Y. Xu, "Multi-pair two-way massive MIMO AF full-duplex relaying with ZFR/ZFT and imperfect CSI," in 2016 16th ISCIT, Sept 2016, pp. 27-32.

[3] H. Q. Ngo, H. A. Suraweera, M. Matthaiou, and E. G. Larsson, "Multipair full-duplex relaying with massive arrays and linear processing," IEEE J. Sel. Areas Commun., vol. 32, no. 9, pp. 1721-1737, Sept 2014.

[4] C. Kong et al., "Multipair two-way half-duplex DF relaying with massive arrays and imperfect CSI," IEEE Trans. Wireless Commun., vol. 17, no. 5, pp. 3269-3283, May 2018.

[5] F. Tan, T. Lv, and S. Yang, "Power allocation optimization for energyefficient massive MIMO aided multi-pair decode-and-forward relay systems," IEEE Trans. Commun., vol. 65, no. 6, pp. 2368-2381, June 2017.

[6] H. Cui, L. Song, and B. Jiao, "Multi-pair two-way amplify-and-forward relaying with very large number of relay antennas," IEEE Trans. Wireless Commun., vol. 13, no. 5, pp. 2636-2645, May 2014.

[7] S. J. Kim, N. Devroye, P. Mitran, and V. Tarokh, "Achievable rate regions and performance comparison of half duplex bi-directional relaying protocols," IEEE Trans. Inf. Theory, vol. 57, no. 10, pp. 6405-6418, Oct 2011.

[8] C. Masouros and M. Matthaiou, "Space-constrained massive MIMO: Hitting the wall of favorable propagation," IEEE Commun. Lett., vol. 19, no. 5, pp. 771-774, May 2015.

[9] S. Biswas, C. Masouros, and T. Ratnarajah, "Performance analysis of large multiuser MIMO systems with space-constrained 2-D antenna arrays," IEEE Trans. Wireless Commun., vol. 15, no. 5, pp. 3492-3505, May 2016.

[10] P. V. Amadori and C. Masouros, "Large scale antenna selection and precoding for interference exploitation," IEEE Trans. Commun., vol. 65, no. 10, pp. 4529-4542, Oct 2017.

[11] H. Q. Ngo, E. G. Larsson, and T. L. Marzetta, "Massive MU-MIMO downlink TDD systems with linear precoding and downlink pilots," in 2013 51st Allerton, Oct 2013, pp. 293-298. 
[12] A. Garcia-Rodriguez, C. Masouros, and P. Rulikowski, "Reduced switching connectivity for large scale antenna selection," IEEE Trans. Commun., vol. 65, no. 5, pp. 2250-2263, May 2017.

[13] N. Fatema, G. Hua, Y. Xiang, D. Peng, and I. Natgunanathan, "Massive MIMO linear precoding: A survey," IEEE Systems Journal, pp. 1-12, 2018.

[14] J. Qian, C. Masouros, and A. Garcia-Rodriguez, "Partial csi acquisition for size-constrained massive mimo systems with user mobility," IEEE Trans. Veh. Tech., vol. 67, no. 9, pp. 9016-9020, Sep. 2018.

[15] A. Garcia-Rodriguez and C. Masouros, "Exploiting the increasing correlation of space constrained massive MIMO for CSI relaxation," IEEE Trans. Commun., vol. 64, no. 4, pp. 1572-1587, April 2016.

[16] C. Masouros, M. Sellathurai, and T. Ratnarajah, "Large-scale MIMO transmitters in fixed physical spaces: The effect of transmit correlation and mutual coupling," IEEE Trans. Commun., vol. 61, no. 7, pp. 2794 2804, July 2013.

[17] H. Yu, Y. Li, X. Zhong, L. Wang, and J. Wang, "The analysis of the energy efficiency for the decode-and-forward two-way relay networks,' in 2013 IEEE WCNC, April 2013, pp. 2823-2827.

[18] Z. Zhang, Z. Chen, M. Shen, and B. Xia, "Spectral and energy efficiency of multipair two-way full-duplex relay systems with massive MIMO," IEEE J. Sel. Areas Commun., vol. 34, no. 4, pp. 848-863, April 2016.

[19] N. Shariati, E. Bjrnson, M. Bengtsson, and M. Debbah, "Lowcomplexity polynomial channel estimation in large-scale MIMO with arbitrary statistics," IEEE J. Sel. Topics Signal Process., vol. 8, no. 5, pp. 815-830, Oct 2014.

[20] B. Rankov and A. Wittneben, "Spectral efficient protocols for halfduplex fading relay channels," IEEE J. Sel. Areas Commun., vol. 25, no. 2, pp. 379-389, February 2007.

[21] Q. Zhang, S. Jin, K. Wong, H. Zhu, and M. Matthaiou, "Power scaling of uplink massive MIMO systems with arbitrary-rank channel means," IEEE J. Sel. Topics Signal Process., vol. 8, no. 5, pp. 966-981, Oct 2014.

[22] P. V. Amadori and C. Masouros, "Low RF-complexity millimeter-wave beamspace-MIMO systems by beam selection," IEEE Trans. Commun. vol. 63, no. 6, pp. 2212-2223, June 2015. 